\title{
Apoio paterno ao aleitamento materno: uma revisão integrativa
}

\author{
Fathers support on breastfeeding: an integrative review
}

Bruna Turaça Silva ${ }^{1}$, Luciano Borges Santiago ${ }^{2}$, Joel Alves Lamonier ${ }^{3}$

\section{RESUMO}

Objetivo: Identificar, na literatura científica, publicações sobre a participação do pai ou companheiro no aleitamento materno.

Fontes de dados: Realizou-se uma revisão integrativa no período de 1995 a 2010, utilizando-se os unitermos "pai" e "aleitamento materno" nas bases de dados LILACS, SciELO, BDENF e PubMed/MEDLINE. Os dados obtidos foram organizados em três categorias: o pai como suporte para a amamentação; percepções paternas sobre a amamentação; e o impacto da intervenção educativa sobre aleitamento para os pais.

Síntese dos dados: Foram identificadas 44 publicações que mostraram que o apoio social, profissional e familiar foi imprescindível para o sucesso do aleitamento materno. $\mathrm{O}$ pai foi destacado como suporte fundamental pela forte influência na decisão da mulher em amamentar e na sua continuidade. Contudo, a participação do pai exibe sentimentos ambivalentes: competitividade com a mãe vs. proteção; exclusão vs. aumento do vínculo familiar; apoio vs. preconceitos. Os profissionais de saúde, apontados como referência na busca de informações, mostram-se despreparados para atender aos pais.

Conclusões: Foram encontradas várias produções científicas ressaltando a relevância do apoio paterno para o sucesso do aleitamento materno. Contudo, a maioria das pesquisas apresenta abordagem descritiva, havendo poucos estudos com intervenções educativas.

Palavras-chave: aleitamento materno; pai; apoio social.

\section{ABSTRACT}

Objective: To identify publications regarding the role of the father or partner in the breastfeeding practice.

Data source: An integrative review of the literature was performed searching for articles published between 1995 and 2010, using the key-words "father" and "breastfeeding" in following databases: LILACS, SciELO, BDENF e PubMed/ MEDLINE. Data were organized in three categories: the supporting role of the father on breastfeeding; paternal perceptions about breastfeeding; and the impact of educational intervention on breastfeeding for parents.

Data synthesis: 44 articles showed that social, professional and family support is vital to successful breastfeeding. The father was highlighted as crucial supporter due to his strong influence over women's decision to breastfeed and to maintain breastfeeding. However, the involvement of the father presents evasive patterns: competition with the mother vs. protection, exclusion vs. increase strength in the family relationships, support vs. prejudices. Health professionals were reported as reference sources of education, however they were shown as unprepared to assist the parents.

Conclusions: Several studies on the theme of paternal support were found in the literature, which highlights its importance for successful breastfeeding. However, most of the research had a descriptive approach and there were few studies on educational interventions.

Key-words: breastfeeding; fathers; social support.
Instituição: Universidade Federal do Triângulo Mineiro (UFTM), Uberaba, MG, Brasil

${ }^{1}$ Bacharel em Enfermagem pela UFTM, Uberaba, MG, Brasil

2Doutor em Pediatria pela Faculdade de Medicina de Ribeirão Preto da Universidade de São Paulo, Ribeirão Preto, SP, Brasil

${ }^{3}$ Professor Titular de Pediatria da Faculdade de Medicina da Universidade Federal de Minas Gerais, Belo Horizonte, MG, Brasil
Endereço para correspondência:

Bruna Turaça Silva

Rua Paraná, 1.051, apto 201 - Bairro Brasil

CEP 38400-654 - Uberlândia /MG

E-mail: brunekinha1@hotmail.com

Conflito de interesse: nada a declarar

Recebido em: 22/2/2011

Aprovado em: 27/6/2011 


\section{Introdução}

No Brasil, a partir da década de 1980, houve um grande avanço a favor da amamentação. O Programa Nacional de Incentivo ao Aleitamento Materno (PNIAM), juntamente com órgãos internacionais (Fundo das Nações Unidas para a Infância - UNICEF - e Organização Mundial da Saúde - OMS), algumas organizações não-governamentais e a Sociedade Brasileira de Pediatria contribuíram para o aumento da duração e da taxa de aleitamento natural. De acordo com a última pesquisa nacional de 2008 , a probabilidade de crianças menores do que um ano estarem em aleitamento materno exclusivo foi de $23,3 \%$ aos quatro meses e $9,5 \%$ aos seis meses ${ }^{(1)}$. Tais dados evidenciam que uma minoria segue o preconizado pela OMS e pelo Ministério da Saúde (MS), que recomendam oferecer aleitamento materno exclusivo até os seis meses, complementado até dois anos de idade ou mais ${ }^{(2)}$.

O ser humano é um mamífero da classe de animais vertebrados, caracterizados pela presença de glândulas mamárias nas fêmeas que produzem leite para alimentação dos filhotes. Portanto, o ato de amamentar deveria ser a regra e não a exceção. Contudo, apesar de ser biologicamente determinada, no ser humano a amamentação sofre fortes influências socioculturais ${ }^{(3)}$. Na tentativa de incentivar a amamentação, muitas campanhas trazem como slogan "amamentar é um ato de amor" e, na lógica descrita anteriormente, prover alimento aos filhos é possível apenas para as mulheres. Neste contexto, a mãe fica sendo a única responsável pelo sucesso do aleitamento materno e o não aleitamento ou o desmame precoce associam-na como corresponsável por possíveis agravos na saúde de seu filho. Esse tipo de abordagem pode ser prejudicial, uma vez que impõe exclusivamente à mãe um fardo que deveria ser dividido com seu companheiro, familiares e profissionais de saúde $e^{(4)}$.

$\mathrm{O}$ ato de amamentar é uma tarefa difícil para muitas mulheres ${ }^{(5,6)}$, pois além de todas as dificuldades com o manejo clínico, ainda existe a ansiedade gerada pelo tempo que consideram "perder" ao amamentar ${ }^{(7)}$. Nessa situação, na vida da mulher, o apoio é imprescindível. Mulheres entrevistadas no período puerperal revelaram a necessidade de outra pessoa para ajudar, esclarecer e acompanhar; os familiares e pessoas significativas devem agir como fontes de ajuda, e os profissionais de saúde, principalmente os de enfermagem e pediatras, como fontes de informação( ${ }^{(8)}$.

Quanto à ajuda familiar, destacam-se como entes mais próximos: a mãe da puérpera e o pai do recém-nascido. $\mathrm{O}$ apoio paterno é um importante aliado do aleitamento. O homem, enquanto pai e companheiro, deve participar da saúde integral da mulher e da criança. Um estudo exploratório descritivo de abordagem qualitativa, ao verificar a opinião do pai, concluiu serem todos os participantes a favor da amamentação, por trazer benefícios tanto para o bebê quanto para a mãe $e^{(9)}$. Quando indagados sobre as mudanças ocorridas na vida conjugal, os pais consideram que o ato de amamentar demanda maior dedicação da mulher, refletindo nos afazeres diários e horários de descanso, porém compreendem a sua importância e se comprometem em apoiar ${ }^{(10)}$. Contudo, a amamentação ainda é, para alguns pais, uma ação centrada no corpo biológico e, consequentemente, pertence apenas à mulher, apoiando a mulher não como pais auxiliadores, mas como pais provedores do lar ${ }^{(11)}$.

De acordo com o exposto, é fato que a nutriz necessita de apoio familiar, profissional e social, sendo o pai destacado como o mais importante aliado. A revisão integrativa de literatura pode auxiliar em pesquisas de campo, visto permitir cruzar resultados e considerações de diversos estudos, levando a uma conclusão clara e objetiva acerca de determinado tema ${ }^{(12)}$. Com o intuito de identificar o papel do pai no puerpério, a efetividade do seu apoio, a participação dos profissionais de saúde na sua inclusão, os tipos de metodologias utilizadas para esse tema e a disponibilidade de produções atuais, é que se justifica a presente pesquisa, a qual objetivou identificar produções científicas que discorram sobre a participação do pai no aleitamento materno.

\section{Método}

Trata-se de uma revisão integrativa cuja trajetória metodológica percorrida apóia-se na leitura exploratória e seletiva do material de pesquisa, contribuindo para o processo de síntese e análise dos resultados de vários estudos, criando-se, assim, um enfoque literário claro e objetivo.

Foi realizada busca bibliográfica nos bancos de dados Literatura Latino-Americana e do Caribe (LILACS), Literatura Internacional em Ciências da Saúde e Biomédica (PubMed/ MEDLINE), Base de Dados de Enfermagem (BDENF) e Biblioteca Científica Eletrônica Online (SciELO), utilizando-se os unitermos "aleitamento materno" como descritor do artigo e "pai" como palavra em todo texto. A revisão compreendeu o período de 1995 a 2010.

Foram encontrados vários artigos que relacionavam pai e aleitamento materno, sendo necessário lançar mão de critérios de inclusão para garantir a temática, como apoio paterno na 
amamentação, significando ter o pai como foco principal em pelo menos um dos objetivos e/ou conter resultados e/ou conclusões a respeito do apoio paterno para a amamentação. Primeiramente, analisaram-se títulos e resumos. Após constatar a existência destes critérios, passou-se à leitura na íntegra. A revisão constou de 44 publicações, três monografias, 38 artigos científicos e três capítulos de livros.

\section{Resultados e Discussão}

A distribuição das publicações ao longo dos 15 anos estudados foi uniforme, destacando-se o ano de 2006, com o maior número de produções. No ano de 2010, estavam disponíveis quatro publicações, das quais três eram descritivas e uma era revisão de literatura. Quanto ao idioma, verifica-se que a língua inglesa foi a mais frequente $(59,0 \%)$, seguida das línguas portuguesa $(36,0 \%)$ e espanhola $(4,5 \%)$. A pequena quantidade de artigos disponíveis na língua portuguesa pode dificultar o acesso a estas informações em nosso país e prejudicar a assistência que deve ser sempre baseada em evidências.

Quanto à metodologia, observou-se predomínio da abordagem quantitativa (48\%) sobre a qualitativa (38\%) e a revisão bibliográfica (9\%); apenas dois eram estudos randomizados. Estudos quantitativos contribuem com dados concretos e os qualitativos conseguem abordar bem o enfoque psicológico pertinente à temática. Portanto, o uso das duas linhas metodológicas em uma mesma pesquisa a torna completa. A reduzida quantidade de estudos de intervenção é preocupante, pois são estes que registram a eficácia ou não das ações e norteiam a atuação dos profissionais de saúde.

Os dados obtidos foram organizados em três categorias: o pai como suporte para a amamentação (24 publicações); percepções paternas sobre a amamentação (15 publicações) e o impacto da intervenção educativa sobre aleitamento para pais (cinco publicações).

O pai como suporte para a amamentação (Quadro 1)

Os estudos nos mostraram a realidade de diversos países. Considerando ser a amamentação um híbrido entre a natureza e a cultura, a divergência de costumes deveria fazer muita diferença quanto à necessidade de apoio durante a amamentação. Entretanto, apesar das especificidades de cada povo, o suporte para o sucesso do aleitamento materno mostrou ser indispensável em todos.

Apesar de a revisão buscar especificamente o apoio do pai, os estudos não deixam de abordar o amparo à puérpera por outros atores, como a avó materna, os profissionais de saúde e a sociedade de modo geral. A avó materna é referida pelas mães como essencial, embora algumas atitudes, por falta de conhecimento e sem intenção, possam provocar o desmame precoce $^{(13)}$. A sociedade representa um paradoxo, ora age com campanhas em apoio ao aleitamento, ora a bicos artificiais, sendo por muitas vezes interpretada como preconceituosa à prática milenar da amamentação ${ }^{(14)}$. O profissional de saúde é sempre apontado como fonte de informação e, devido a esta confiança, deve-se investir em capacitações visando uma assistência adequada e com embasamento científico ${ }^{(15)}$.

Quadro 1 - Estudos clínicos e epidemiológicos sobre o apoio paterno

\begin{tabular}{|c|c|c|c|}
\hline & Método & Objetivos & Considerações finais do artigo \\
\hline $\begin{array}{l}\text { Humphereys } \\
\text { et } a^{\beta 0}\end{array}$ & $\begin{array}{l}\text { Transversal; quantitativo; } \\
\text { entrevista com gestantes }\end{array}$ & $\begin{array}{l}\text { Relacionar intenção de } \\
\text { amamentar com apoio social }\end{array}$ & $\begin{array}{l}\text { O apoio de profissionais, familiares } \\
\text { e do pai contribui para o } \mathrm{AM}\end{array}$ \\
\hline Scott et $a R^{21}$ & $\begin{array}{l}\text { Prospectivo; quantitativo; } \\
\text { entrevista com as mães }\end{array}$ & $\begin{array}{l}\text { Investigar os fatores associados } \\
\text { ao AM }\end{array}$ & $\begin{array}{l}\text { Pai desempregado e indiferente ao } \\
\text { AM são fatores negativos }\end{array}$ \\
\hline Kuan et $a{ }^{47}$ & $\begin{array}{l}\text { Prospectivo; qualitativo; } \\
\text { entrevista com puérperas }\end{array}$ & $\begin{array}{l}\text { Identificar fatores do sistema de } \\
\text { saúde que promovem o AM }\end{array}$ & $\begin{array}{l}\text { Inclusão dos pais e familiares } \\
\text { através de visitas domiciliares }\end{array}$ \\
\hline $\begin{array}{l}\text { Shepherd } \\
\text { et } a l^{41}\end{array}$ & Longitudinal; quantitativo & $\begin{array}{l}\text { Comparar condições sociais } \\
\text { com a alimentação infantil }\end{array}$ & $\begin{array}{l}\text { O AM é menor em condições } \\
\text { socioeconômicas desfavoráveis }\end{array}$ \\
\hline Barriuso et al ${ }^{17}$ & $\begin{array}{l}\text { Prospectivo; quantitativo; } \\
\text { entrevista com as mães }\end{array}$ & $\begin{array}{l}\text { Investigar fatores que influem } \\
\text { nas taxas de AM }\end{array}$ & $\begin{array}{l}\text { A presença do pai não influenciou. } \\
\text { Cesária e retardo da } 1^{a} \text { mamada } \\
\text { foram fatores negativos }\end{array}$ \\
\hline
\end{tabular}


Quadro 1 - Continuação

\begin{tabular}{|c|c|c|c|}
\hline Arora et $a^{24}$ & $\begin{array}{l}\text { Prospectivo; quantitativo; } \\
\text { entrevista com as mães }\end{array}$ & $\begin{array}{l}\text { Determinar o que influencia } \\
\text { a alimentação infantil e a } \\
\text { duração do AM }\end{array}$ & $\begin{array}{l}\text { Orientações no pré-natal influem } \\
\text { na escolha; ação do pai, pouco } \\
\text { leite e volta ao trabalho na duração }\end{array}$ \\
\hline $\begin{array}{l}\text { Ichisato e } \\
\text { Shimo }^{4}\end{array}$ & Revisão bibliográfica & $\begin{array}{l}\text { Revisitar o desmame precoce } \\
\text { através de recortes históricos }\end{array}$ & $\begin{array}{l}\text { O AM deve ultrapassar o binômio } \\
\text { mãe-filho, pois a visão do homem/ } \\
\text { pai também é importante }\end{array}$ \\
\hline Earle $^{43}$ & $\begin{array}{l}\text { Transversal; qualitativo; } \\
\text { entrevista com as mães }\end{array}$ & $\begin{array}{l}\text { Explorar experiências e } \\
\text { percepções de mães sobre AM }\end{array}$ & $\begin{array}{l}\text { O desejo de voltar a ser um } \\
\text { "ser individual" é fator para não } \\
\text { amamentar }\end{array}$ \\
\hline Khoury et a ${ }^{44}$ & $\begin{array}{l}\text { Randomizado; quantitativo; } \\
\text { entrevista antes e após } \\
\text { vídeo }\end{array}$ & $\begin{array}{l}\text { Avaliar a efetividade de } \\
\text { programa de promoção do AM }\end{array}$ & $\begin{array}{l}\text { As mães se sensibilizaram para a } \\
\text { participação do pai no AM }\end{array}$ \\
\hline $\begin{array}{l}\text { Ramos e } \\
\text { Almeida }^{5}\end{array}$ & $\begin{array}{l}\text { Estudo transversal; } \\
\text { abordagem qualitativa; } \\
\text { entrevista com as mães }\end{array}$ & $\begin{array}{l}\text { Compreender motivos para o } \\
\text { desmame }\end{array}$ & $\begin{array}{l}\text { A falta de apoio paterno e de } \\
\text { familiares levou à vulnerabilidade } \\
\text { emocional }\end{array}$ \\
\hline Ludvigsson $^{18}$ & $\begin{array}{l}\text { Retrospectivo; quantitativo; } \\
\text { entrevista com as mães }\end{array}$ & $\begin{array}{l}\text { Relacionar opiniões sobre AM } \\
\text { com padrão alimentar de RN }\end{array}$ & $\begin{array}{l}\text { As opiniões não coincidiram com o } \\
\text { padrão alimentar }\end{array}$ \\
\hline Kong e Lee ${ }^{31}$ & $\begin{array}{l}\text { Prospectivo; quantitativo e } \\
\text { qualitativo; entrevista com } \\
\text { primíparas }\end{array}$ & $\begin{array}{l}\text { Investigar conhecimentos sobre } \\
\text { AM e os fatores que influem na } \\
\text { decisão de amamentar }\end{array}$ & $\begin{array}{l}\text { Acreditam em mitos e apontam o } \\
\text { apoio paterno como fator decisivo }\end{array}$ \\
\hline $\begin{array}{l}\text { Silveira e } \\
\text { Lamounier }^{20}\end{array}$ & $\begin{array}{l}\text { Retrospectivo; quantitativo; } \\
\text { entrevista com as mães }\end{array}$ & $\begin{array}{l}\text { Identificar variáveis associadas } \\
\text { à duração do AM em municípios } \\
\text { de Minas Gerais }\end{array}$ & $\begin{array}{l}\text { Escolaridade paterna; uso de } \\
\text { chupeta e pai não residir com a } \\
\text { criança }\end{array}$ \\
\hline $\mathrm{Hoga}^{29}$ & Capítulo de livro & & $\begin{array}{l}\text { Há gradativo aumento da } \\
\text { responsabilidade masculina e } \\
\text { inclusão na saúde da mulher }\end{array}$ \\
\hline $\begin{array}{l}\text { Rudman e } \\
\text { Waldenström }\end{array}$ & $\begin{array}{l}\text { Prospectivo; qualitativo; } \\
\text { entrevista com as mães }\end{array}$ & $\begin{array}{l}\text { Descrever experiências } \\
\text { negativas de puérperas no } \\
\text { pós-parto }\end{array}$ & $\begin{array}{l}\text { Falta de informação } \\
\text { individualizada, adequada gestão } \\
\text { dos sintomas, apoio ao AM e } \\
\text { inclusão paterna }\end{array}$ \\
\hline $\begin{array}{l}\text { Clifford e } \\
\text { Mclntyre }\end{array}$ & e literatura & $\begin{array}{l}\text { Identificar quem sustenta } \\
\text { (apóia) o AM }\end{array}$ & $\begin{array}{l}\text { Pais, familiares, profissionais e } \\
\text { amigos apóiam. Empregadores e a } \\
\text { sociedade não apóiam efetivamente }\end{array}$ \\
\hline $\begin{array}{l}\text { Bottaro e } \\
\text { Giugliani }^{23}\end{array}$ & $\begin{array}{l}\text { Transversal; quantitativa; } \\
\text { questionário com alunos }\end{array}$ & $\begin{array}{l}\text { Avaliar conhecimento, } \\
\text { percepções, e vivências de } \\
\text { alunos do ensino médio }\end{array}$ & $\begin{array}{l}100 \% \text { consideraram importante o } \\
\text { apoio do pai, mas } 70 \% \text { acham que } \\
\text { estes ajudam dando mamadeira. }\end{array}$ \\
\hline $\begin{array}{l}\text { Mota e } \\
\text { Gomes }^{19}\end{array}$ & $\begin{array}{l}\text { Transversal; quantitativo; } \\
\text { questionário com os pais }\end{array}$ & $\begin{array}{l}\text { Caracterizar conhecimentos, } \\
\text { concepções e a participação de } \\
\text { homens no AM }\end{array}$ & $\begin{array}{l}\text { Os participantes possuem algum } \\
\text { conhecimento sobre o tema, mas } \\
\text { se confundem em muitos aspectos }\end{array}$ \\
\hline Marques et al' & Revisão bibliográfica & $\begin{array}{l}\text { Influência da rede social da } \\
\text { lactante na amamentação }\end{array}$ & $\begin{array}{l}\text { Atores principais: pai, avó e } \\
\text { profissionais de saúde }\end{array}$ \\
\hline Paula et aR6 & $\begin{array}{l}\text { Transversal; qualitativo; } \\
\text { entrevista com os pais }\end{array}$ & $\begin{array}{l}\text { Investigar conhecimento, } \\
\text { participação e fontes de } \\
\text { informação dos pais sobre AM }\end{array}$ & $\begin{array}{l}\text { Querem que seus filhos sejam } \\
\text { amamentados, apesar de não ter } \\
\text { recebido orientação profissional }\end{array}$ \\
\hline $\begin{array}{l}\text { Alexander } \\
\text { et } a^{25}\end{array}$ & $\begin{array}{l}\text { Transversal; qualitativo; } \\
\text { entrevista com as mães }\end{array}$ & $\begin{array}{l}\text { Determinar fatores que } \\
\text { influenciam na decisão de afro- } \\
\text { americanas amamentarem. }\end{array}$ & $\begin{array}{l}\text { Amamentam pelos benefícios ao } \\
\text { bebê, e não o fazem por medo da } \\
\text { dor e pela volta ao trabalho }\end{array}$ \\
\hline $\begin{array}{l}\text { Johansson } \\
\text { et } a{ }^{42}\end{array}$ & $\begin{array}{l}\text { Prospectivo; qualitativo; } \\
\text { entrevista com pai e mãe }\end{array}$ & $\begin{array}{l}\text { Compreender as experiências } \\
\text { de pais desde a alta hospitalar } \\
\text { até os cuidados domiciliares }\end{array}$ & $\begin{array}{l}\text { O AM foi principal tema no processo } \\
\text { de aprendizagem e os profissionais } \\
\text { de saúde como suporte }\end{array}$ \\
\hline
\end{tabular}

AM: aleitamento materno; RN: recém-nascido 
Dentre todos os entes familiares e pessoas próximas citadas, a presença do pai é o suporte de maior relevância para a amamentação na perspectiva materna. A influência paterna é destacada como um dos motivos para o aumento da sua incidência e prevalência, ou seja, o pai influi na decisão da mulher de amamentar e contribui para a sua continuidade ${ }^{(16)}$. Apenas dois estudos resultaram em nenhuma relação entre pais e aleitamento materno ${ }^{(17,18)}$. O conhecimento do pai sobre amamentação é imprescindível. Contudo, muitas vezes este ocorre envolto por dúvidas em aspectos distintos ${ }^{(19)}$, evidenciando a necessidade de intervenção profissional.

O desejo de amamentar, na maioria das mulheres, aflora antes da concepção ou no primeiro trimestre da gestação, considerando a influência do pai nesta decisão e a maior prevalência do aleitamento materno nas mulheres casadas ou com relacionamento estável, pode-se inferir que há uma relação com a presença cotidiana do pai/companheiro ${ }^{(20)}$.

O sucesso do aleitamento, contudo, não depende somente da sua presença, mas também da sua atitude. Existe o pai do tipo atuante, que tem taxa de aleitamento maior que o pai do tipo indiferente ${ }^{(21)}$. A atuação do pai ainda é permeada por incertezas e dificuldades, ao ponto de crianças do ensino fundamental e até mesmo algumas mães relatarem que a inclusão do pai na alimentação infantil se dá através da mamadeira ${ }^{(22,23)}$.

O apoio financeiro, apesar de não ser uma expectativa declarada das puérperas, representa um suporte indireto, pois o desemprego paterno culmina na volta antecipada da mulher ao trabalho ${ }^{(24,25)}$.

De acordo com a Constituição Brasileira é direito de mulheres e homens tomar uma decisão livre sobre as práticas relativas à sexualidade e à reprodução. Também, é reconhecida a igualdade de direitos e obrigações de pais e mães na criação dos filhos. A prática de amamentar deve ser centralizada na conjugalidade de todos os membros da família, o que direciona para a formulação de políticas públicas de saúde que visem inserir a família, principalmente o pai, no pré-natal e na atenção à saúde materno infantil como forma de articular e traçar objetivos em comum nas ações dos profissionais ${ }^{(26)}$.

\section{Percepções paternas sobre a amamentação (Quadro 2)}

O enfoque materno-infantil, em geral restrito ao binômio mãe-filho, tem ampliado sua temática ao incluir assuntos como relações sociais de gênero. $\mathrm{O}$ mundo masculino é explorado no âmbito psicológico e da saúde e, considerando a premissa de que a melhoria dos indicadores de saúde materno infantil está intimamente ligada à mudança de atitude dos homens; as pesquisas tendem a aumentar cada vez mais ${ }^{(27,28)}$.

O homem associa relação sexual com prazer, enquanto a mulher a associa com reprodução. Isto explica perspectivas tão distintas, o precoce desabrochar do instinto materno e a insegurança do homem frente ao papel de pai ${ }^{(29)}$.

O conhecimento paterno sobre os benefícios da amamentação para o bebê mostra-se vasto ${ }^{(28)}$; somado à influencia que ele exerce sobre sua companheira e ao sentimento de proteção para com o bebê, o pai apresenta grande potencial de tornar-se um suporte para o aleitamento materno ${ }^{(30-32)}$. A mãe, geralmente, não é lembrada como beneficiária e sim como protagonista. Os pais referem-se a elas apenas como provedoras da alimentação do bebê e não mencionam a rápida involução uterina, a redução do risco de câncer de mama, entre outros benefícios do aleitamento materno para a saúde da mãe, os quais parecem ser desconhecidos ou ignorados.

$\mathrm{O}$ fato do ato fisiológico de prover alimento ao filho ser exclusivo da mulher gera nos seus companheiros sentimentos de isolamento e competitividade ${ }^{(26)}$. Devido a isso, o pai direciona sua busca por informações para temas como a interação pai-filho, sendo o aleitamento um dos menos procurados ${ }^{(33)}$.

A amamentação ainda está centrada no corpo biológico ${ }^{(27)}$. Infelizmente, discriminar atributos femininos e masculinos é também parte da cultura da mulher. Não obstante, é possível encontrar companheiros interessados que referem exclusão não apenas na amamentação como também em todo o processo do cuidar ${ }^{(26,34)}$. A aceitação materna do apoio paterno é ponto crucial para que este aconteça.

Da mesma maneira, os profissionais de saúde, que deveriam facilitar e até mesmo buscar ativamente a inclusão paterna no ciclo gravídico puerperal, transmitem inaptidão para atuar com pais ${ }^{(35)}$. Pais de crianças entre um e 12 meses relataram não terem sido solicitados pelos profissionais no pré-natal, apesar de estarem presentes no serviço de saúde ${ }^{(36)}$. Ainda que tenham a intenção de apoiar, encontram dificuldades, como horários das consultas e grupos de gestantes incompatíveis com os de seu trabalho ${ }^{(37,38)}$.

A vivência do pai acerca do aleitamento materno é permeada por sentimentos paradoxais. Sentem-se felizes e querem apoiar, simultaneamente sentem-se frustrados e excluídos. Acreditam que a amamentação representa o vínculo afetivo, contudo minimiza sua participação nos cuidados com o bebê. Além disso, enfatizam que pode interferir na sexualidade do casal ${ }^{(39)}$. Esta ambivalência se relaciona com o medo do desconhecido: a transformação do núcleo familiar. 
Quadro 2 - Estudos clínicos e epidemiológicos sobre as perspectivas paternas

\begin{tabular}{|c|c|c|c|}
\hline & Método & Objetivos & Considerações finais do artigo \\
\hline Aquino et $a^{27}$ & Capítulo de livro & & $\begin{array}{l}\text { O Brasil tende para a inclusão } \\
\text { paterna cada vez mais intensa }\end{array}$ \\
\hline Arilha ${ }^{28}$ & Capítulo de livro & & $\begin{array}{l}\text { Questões de gênero são uma } \\
\text { barreira para a inclusão paterna }\end{array}$ \\
\hline $\begin{array}{l}\text { Molina e } \\
\text { Rojas }^{39}\end{array}$ & $\begin{array}{l}\text { Transversal; qualitativo; } \\
\text { entrevista com pais }\end{array}$ & $\begin{array}{l}\text { Descrever o pai como agente } \\
\text { de suporte e proteção do AM }\end{array}$ & $\begin{array}{l}\text { O AM é benéfico para o bebê, mas } \\
\text { interfere na atividade sexual }\end{array}$ \\
\hline $\begin{array}{l}\text { Serafim e } \\
\text { Lindsey }^{36}\end{array}$ & $\begin{array}{l}\text { Transversal; quantitativo; } \\
\text { entrevista com os pais }\end{array}$ & $\begin{array}{l}\text { Identificar conhecimento e } \\
\text { envolvimento do pai }\end{array}$ & $\begin{array}{l}25 \% \text { participaram do pré-natal. } \\
\text { A maioria demonstrou pouco } \\
\text { conhecimento }\end{array}$ \\
\hline Fernandes ${ }^{34}$ & $\begin{array}{l}\text { Transversal; qualitativo; } \\
\text { entrevista com os pais }\end{array}$ & $\begin{array}{l}\text { Identificar os fatores que } \\
\text { permeiam a vivência do pai } \\
\text { durante o AM }\end{array}$ & $\begin{array}{l}\text { A vivência dos pais é permeada } \\
\text { por fatores de ordem econômica, } \\
\text { conjugal e prática }\end{array}$ \\
\hline $\begin{array}{l}\text { Stremler e } \\
\text { Lovera }^{50}\end{array}$ & $\begin{array}{l}\text { Transversal, quantitativo, } \\
\text { entrevista com pais }\end{array}$ & $\begin{array}{l}\text { Descrever programa de } \\
\text { capacitação sobre AM para pais }\end{array}$ & $\begin{array}{l}\text { Classificaram como muito bom, } \\
\text { pois capacitam multiplicadores }\end{array}$ \\
\hline $\begin{array}{l}\text { Ingram e } \\
\text { Johnson }^{13}\end{array}$ & $\begin{array}{l}\text { Prospectivo; qualitativo; } \\
\text { entrevista com pais e avós }\end{array}$ & $\begin{array}{l}\text { Avaliar o conhecimento e } \\
\text { capacidade de apoiar o AM }\end{array}$ & $\begin{array}{l}\text { Avós: têm conhecimento } \\
\text { retrógrado. Pais: não participam do } \\
\text { pré-natal }\end{array}$ \\
\hline Brito et $\left.a\right|^{40}$ & $\begin{array}{l}\text { Transversal; quantitativo; } \\
\text { entrevista com os pais }\end{array}$ & $\begin{array}{l}\text { Verificar a concepção do } \\
\text { pai sobre o AM e suas } \\
\text { contribuições no processo de } \\
\text { lactação }\end{array}$ & $\begin{array}{l}16 \% \text { admitiram não incentivar o } \\
\text { AM; dos que incentivam } 17 \% \text { o } \\
\text { fazem por imposição }\end{array}$ \\
\hline $\begin{array}{l}\text { Garfield e } \\
\text { Isacco }^{37}\end{array}$ & $\begin{array}{l}\text { Transversal; qualitativo; } \\
\text { entrevista com os pais }\end{array}$ & $\begin{array}{l}\text { Investigar o envolvimento dos } \\
\text { pais em um sistema de saúde }\end{array}$ & $\begin{array}{l}\text { Sentem-se excluídos pelos } \\
\text { profissionais }\end{array}$ \\
\hline $\begin{array}{l}\text { Brito e } \\
\text { Oliveira }^{9}\end{array}$ & $\begin{array}{l}\text { Transversal; qualitativo; } \\
\text { entrevista com os pais }\end{array}$ & $\begin{array}{l}\text { Verificar a opinião do pai acerca } \\
\text { do AM }\end{array}$ & $\begin{array}{l}\text { Demonstram opiniões positivas } \\
\text { e conhecimento sobre seus } \\
\text { benefícios }\end{array}$ \\
\hline $\begin{array}{l}\text { Brito e } \\
\text { Oliveira }^{10}\end{array}$ & $\begin{array}{l}\text { Transversal; qualitativo; } \\
\text { entrevista com os pais }\end{array}$ & $\begin{array}{l}\text { Identificar as mudanças } \\
\text { ocorridas no convívio conjugal } \\
\text { com o AM }\end{array}$ & $\begin{array}{l}\text { Expressão competitividade e } \\
\text { isolamento em relação à puérpera }\end{array}$ \\
\hline Fletcher et $a^{\beta 3}$ & $\begin{array}{l}\text { Transversal; quantitativo; } \\
\text { questionário para os pais }\end{array}$ & $\begin{array}{l}\text { Avaliar a prontidão dos pais } \\
\text { para utilizar as informações } \\
\text { eletrônicas adaptadas ao papel } \\
\text { do pai }\end{array}$ & $\begin{array}{l}\text { O tópico mais visitado foi interação } \\
\text { pai-filho e os menos visitados } \\
\text { foram AM e relação sexual no } \\
\text { pós-parto }\end{array}$ \\
\hline Costa $^{38}$ & $\begin{array}{l}\text { Transversal; qualitativo; } \\
\text { entrevista com os pais }\end{array}$ & $\begin{array}{l}\text { Compreender a representação } \\
\text { do papel do pai no AM e } \\
\text { conhecer facilitadores e } \\
\text { dificultadores }\end{array}$ & $\begin{array}{l}\text { Dificultadores: elevada carga } \\
\text { horária de trabalho e falta de } \\
\text { investimento em atividades } \\
\text { educativas }\end{array}$ \\
\hline Pontes et a/11 & $\begin{array}{l}\text { Transversal; qualitativo; } \\
\text { entrevista com pai e mãe }\end{array}$ & $\begin{array}{l}\text { Identificar sentimentos do pai } \\
\text { durante o AM }\end{array}$ & $\begin{array}{l}\text { A participação do pai está centrada } \\
\text { no corpo biológico }\end{array}$ \\
\hline Tohotoa et $a^{\beta 2}$ & $\begin{array}{l}\text { Transversal; qualitativo; } \\
\text { entrevista com pai e mãe }\end{array}$ & $\begin{array}{l}\text { Identificar percepções sobre o } \\
\text { apoio paterno ao AM }\end{array}$ & $\begin{array}{l}\text { Para as mães é incentivador e os } \\
\text { pais sentem-se desinformados }\end{array}$ \\
\hline
\end{tabular}

AM: aleitamento materno

Como dito anteriormente, o pai representa uma influência ímpar na decisão da mulher de amamentar. Entretanto, sua participação na amamentação, até o momento, é permeada por dúvidas, preconceitos e até imposição ${ }^{(40)}$. Julgam serem capazes de apoiar apenas por meio de verbalizações positivas e não com ações e mostram-se preconceituosos em relação à exposição pública durante a amamentação ${ }^{(41)}$.

No pós-parto, o desconforto e a insegurança presentes na maioria dos relacionamentos conjugais são mais intensos nos primeiros três meses e podem ser amenizados se discutidos no pré-natal ${ }^{(21)}$. 


\section{O impacto da intervenção educativa sobre aleitamento para pais (Quadro 3)}

A saúde feminina engloba também o ciclo gravídicopuerperal e, dentre tantos assuntos, o aleitamento é descrito por primíparas e seus companheiros como tema principal no processo de aprendizagem ${ }^{(42)}$. A inclusão paterna nos projetos de educação em saúde e de assistência foi recomendada na Conferência Mundial sobre a Mulher em 1995, na cidade de Beijing. A participação do pai é um desafio em foco no mundo todo.

Os profissionais da saúde são referências para quem necessita de informações. Orientações técnicas realizadas no pré-natal, na maternidade ou mesmo no puerpério refletem positivamente nas taxas de incidência e prevalência da amamentação. Para minimizar a ansiedade dos pais, é imprescindível a participação de ambos, pais e mães, em grupos antes restritos às gestantes ${ }^{(29,31,34,43)}$.

Contudo, devido à grande divergência cultural e biológica, a abordagem deve levar em conta características de cada gênero ${ }^{(26)}$. Com os pais, deve haver cautela uma vez que a atitude de imposição paterna, ou seja, a pressão para que a companheira amamente, provoca sentimentos negativos na mulher. Já as mulheres precisam ser sensibilizadas quanto à participação do pai, visando sua concessão e consequente abertura para o processo do cuidar ${ }^{(44)}$. Vale lembrar que a efetividade de uma educação em saúde bem sucedida depende do respeito à especificidade de cada casal.
Muitos estudos associam o nível de escolaridade paterna com o sucesso do aleitamento materno ${ }^{(35,40,45,46)}$. Atentar para a adequação dos materiais didáticos e da linguagem utilizados é crucial para haver melhor compreensão. O acompanhamento domiciliar no puerpério é um exemplo de estratégia individualizada que possibilita uma relação íntima com a puérpera e a inclusão dos pais que não frequentaram o serviço de saúde ${ }^{(47)}$.

Destaca-se que os objetivos da inclusão masculina não devem ter como sujeito exclusivamente a saúde materna e infantil; o homem em si, e não apenas como pai e companheiro, deve também ser considerado ${ }^{(43)}$. A avaliação de grupos de orientação realizados com pais mostra que há aproveitamento, interesse e validação dos pais para com a iniciativa, além de comprovar sua viabilidade ${ }^{(48)}$.

A eficácia do apoio paterno e da educação em saúde para pais tem se afirmado com o resultado de estudos randomizados. A incidência de aleitamento materno nos grupos de estudo que recebem orientações sobre este assunto é maior do que nos grupos controle, que recebem orientações apenas quanto ao cuidado com o bebê $\hat{e}^{(49)}$. A educação em saúde faz diferença na saúde física e mental da família e não requer das instituições ou dos orientadores nada mais do que conhecimento.

Cabe aos profissionais de saúde driblar os empecilhos e buscar vencer o desafio. Quando se planta a semente da informação, se colhe pais multiplicadores. Estes, por

Quadro 3 - Estudos clínicos e epidemiológicos sobre aleitamento materno e educação em saúde acerca do papel paterno

\begin{tabular}{|c|c|c|c|}
\hline & Método & Objetivos & Considerações finais do artigo \\
\hline Cohen et al ${ }^{48}$ & $\begin{array}{l}\text { Transversal; quantitativo; } \\
\text { entrevista com os pais }\end{array}$ & $\begin{array}{l}\text { Descrever um programa de } \\
\text { promoção do AM com funcionários } \\
\text { do sexo masculino }\end{array}$ & $\begin{array}{l}\text { Destaca-se o interesse dos } \\
\text { funcionários e a viabilidade da } \\
\text { educação sobre AM para homens }\end{array}$ \\
\hline Wolfberg et a ${ }^{49}$ & $\begin{array}{l}\text { Randomizado; } \\
\text { quantitativo; entrevista } \\
\text { com pais }\end{array}$ & $\begin{array}{l}\text { Reconhecer a influência do pai na } \\
\text { decisão da mãe em amamentar ou } \\
\text { utilizar fórmula infantil }\end{array}$ & $\begin{array}{l}74 \% \text { das companheiras do grupo de } \\
\text { estudo amamentaram, contra } 41 \% \\
\text { das do grupo controle }\end{array}$ \\
\hline $\begin{array}{l}\text { Alvarado- } \\
\text { Rivera et a/51 }\end{array}$ & $\begin{array}{l}\text { Transversal; quantitativo; } \\
\text { entrevista com os pais }\end{array}$ & $\begin{array}{l}\text { Identificar o conhecimento sobre } \\
\text { AM, as atitudes face à sexualidade } \\
\text { e a disposição para apoiar de } \\
\text { futuros pais }\end{array}$ & $\begin{array}{l}\text { Quanto maior o conhecimento sobre } \\
\text { o AM, maior é a disposição em } \\
\text { apoiá-lo }\end{array}$ \\
\hline $\begin{array}{l}\text { Susin e } \\
\text { Giugliani }{ }^{46}\end{array}$ & $\begin{array}{l}\text { Randomizado; } \\
\text { quantitativo }\end{array}$ & $\begin{array}{l}\text { Avaliar o impacto da inclusão } \\
\text { do pai em um programa de } \\
\text { ensino sobre AM em um hospital } \\
\text { maternidade }\end{array}$ & $\begin{array}{l}\text { A inclusão dos pais aumentou } \\
\text { significativamente as taxas de } \\
\text { AM quando estes possuíam } \\
\text { escolaridade maior que oito anos }\end{array}$ \\
\hline $\begin{array}{l}\text { Iglesias } \\
\text { Casás }^{45}\end{array}$ & $\begin{array}{l}\text { Prospectivo; quantitativo } \\
\text { e qualitativo; entrevista } \\
\text { com as mães }\end{array}$ & $\begin{array}{l}\text { Determinar a prevalência do AM } \\
\text { em Salnés-Espanha e a influência } \\
\text { da rede social e dos profissionais } \\
\text { de saúde no seu início e duração }\end{array}$ & $\begin{array}{l}\text { A escolaridade paterna elevada } \\
\text { foi associada com o início e com a } \\
\text { continuidade do AM }\end{array}$ \\
\hline
\end{tabular}

AM: aleitamento materno 
solidariedade a quem está passando pelas angústias que já foram suas, disseminam o conhecimento a outros pais ${ }^{(50)}$. E quanto maior o conhecimento sobre aleitamento materno, maior é a disposição em apoiá-lo(s1).

\section{Considerações finais}

Evidenciou-se que a mulher no ciclo gravídico puerperal necessita de apoio social, profissional e familiar, sendo o pai o principal suporte. Foram encontradas várias produções científicas acerca da temática na literatura que destacam sua relevância para o sucesso do aleitamento materno. Contudo, a maioria apresenta abordagem descritiva, havendo poucos estudos com intervenções educativas.

A escassez de estudos randomizados leva a pensar que não está havendo intervenções que visem à inclusão paterna na saúde materno-infantil e à educação em saúde sobre aleitamento ou que estas não estão sendo registradas para o conhecimento dos profissionais do meio acadêmico e assistencial.

\section{Referências bibliográficas}

1. Brasil. Ministério da Saúde. Secretaria de Atenção à Saúde-Departamento de Ações Programáticas e Estratégicas. II Pesquisa de prevalência de aleitamento materno nas capitais brasileiras e Distrito Federal. [Série C. Projeto, Programas e Relatórios]. Brasília: Ministério da Saúde, 2009.

2. Brasil. Ministério da Saúde. Secretaria de Políticas Públicas. Organização Pan Americana da Saúde. Guia alimentar para crianças menores de dois anos. Brasília: Ministério da Saúde, 2002.

3. Almeida JA, Novak FR. Breastfeeding: a nature-culture hybrid. J Pediatr (Rio J) 2004;80 (Suppl 5):S119-25.

4. Ichisato SM, Shimo AK. Revisiting early weaning through historical analysis. Rev Latino-Am Enfermagem 2002;10:578-85.

5. Ramos CV, Almeida JA. Breast-feeding: the way it is experienced by women assisted at a pediatrics and maternity hospital in Teresina in the state of Piauí. Rev Bras Saude Mater Infant 2003;3:315-21.

6. Nakano AM. O Aleitamento no cotidiano feminino [tese de mestrado]. Ribeirão Preto: Universidade de São Paulo; 1996.

7. Carvalhaes MA, Corrêa CR. Identification of difficulties at the beginning of breastfeeding by means of protocol application. J Pediatr (Rio J) 2003;79:13-20.

8. Gonçalves AR. Aprender a ser mãe. Processos de aprendizagem de mães primíparas durante os primeiros dois meses pós-parto. Sísifo - Revista de Ciências da Educação 2008;5:59-68.

9. Brito RS, Oliveira EM. Father's opinion concerning breast feeding. Rev Rene 2006;7:9-16.

10. Brito RS, Oliveira EM. Maternal breast-feeding: changes occurred in the father's conjugal life. Rev Gaucha Enferm 2006;27:193-202.

11. Pontes CM, Alexandrino AC, Osório MM. The participation of fathers in the breastfeeding process: experiences, knowledge, behaviors and emotions. J Pediatr (Rio J) 2008;84:357-64.

12. Souza MT, Silva MD, Carvalho R. Integrative review: what is it? How to do it? Einstein 2010;8:102-6

13. Ingram J, Johnson D. A feasibility study of an intervention to enhance family support for breast feeding in a deprived area in Bristol, UK. Midwifery 2004;20:367-79.
A divulgação das ações por meio de publicações científicas é imprescindível para que a prática e o ensino possam ser baseados em evidências.

O homem tem atuado cada vez mais em seu papel de pai, acompanha sua companheira aos serviços de saúde e busca conhecimento a fim de apoiá-la da melhor forma. Em contrapartida, os profissionais de saúde não têm se capacitado para recebê-los na mesma proporção. Durante a graduação, os temas abordados relativos ao aleitamento ainda são, primordialmente, sobre técnica, manejo da amamentação e composição do leite materno, marginalizando os aspectos psicológicos e a inclusão paterna.

Apesar de todas as mudanças em busca da inclusão do homem, este ainda encontra dificuldades para compreender as transformações que ocorrem com as mulheres no decorrer de suas vidas, verdade esta observada e confirmada no cotidiano da assistência. O complexo processo de fusão da atenção à saúde aos diferentes membros de uma família ainda é um desafio a ser vencido.

14. Clifford J, Mclntyre E. Who supports breastfeeding? Breastfeed Rev 2008;16:9-19.

15. Tarkka MT, Paunonen M, Laippala P. Factors related to successful breast feeding by first-time mothers when the child is 3 months old. J Adv Nurs 1999;29:113-8.

16. Marques ES, Cotta RM, Magalhães KA, Sant'Ana LF, Gomes AP, SiqueiraBatista R. The influence of the social net of lactating mothers in the breastfeeding: the strategic role of the relatives and professionals of health. Cienc Saude Colet 2010;15 (Suppl 1):1391-400.

17. Barriuso Lapresa L, Sánchez-Valverde Visus F, Romero Ibarra C, Vitoria Comerzana JC. Hospital guidelines on breastfeeding in the north-center of Spain. An Esp Pediatr 2000;52:225-31.

18. Ludvigsson JF. Breastfeeding in Bolivia - information and attitudes. BMC Pediatr 2003;3:4.

19. Mota E, Gomes CF. O papel do homem no aleitamento materno. Temas Desenvolv 2009;17:22-7.

20. Silveira FJ, Lamounier JA. Factors associated with breastfeeding duration in three cities in the region of Alto Jequitinhonha, Minas Gerais, Brazil. Cad Saude Publica 2006;22:69-77.

21. Scott JA, Aitkin I, Binns CW, Aroni RA. Factors associated with the duration of breastfeeding amongst women in Perth, Australia. Acta Paediatr 1999;88:416-21.

22. Earle S. Why some women do not breast feed: bottle feeding and fathers' role. Midwifery 2000;16:323-30.

23. Bottaro SM, Giugliani ER. Exploratory survey of fifth-grade elementary students in relation to breastfeeding. Cad Saude Publica 2008;24:1599-608.

24. Arora S, McJunkin C, Wehrer J, Kuhn P. Major factors influencing breastfeeding rates: Mother's perception of father's attitude and milk supply. Pediatrics 2000;106:E67.

25. Alexander A, Dowling D, Furman L. What do pregnant low-income women say about breastfeeding? Breastfeed Med 2010;5:17-23.

26. Paula AO, Sartori AL, Martins CA. Breastfeeding: father's orientations, knowledge and participation in the process. Rev Eletr Enf [serial on the Internet] 2010;12:464-70. 
27. Aquino EM, Araújo TV, Marinho LF. Padrões e tendências em saúde reprodutiva no Brasil: bases para uma análise epidemiológica. In: Giffin K, Costa SH, editors. Questões da saúde reprodutiva. Rio de Janeiro: Fiocruz; 1999. p. 187-205.

28. Arilha M. Homens, saúde reprodutiva e gênero: o desafio da inclusão. In: Giffin K, Costa SH, editors. Questões de saúde reprodutiva. Rio de Janeiro: Fiocruz; 1999. p. 455-67.

29. Hoga LA. Perspectiva masculina da saúde da mulher. In: Fernandes RA, Narchi NZ, editors. Enfermagem e saúde da mulher. São Paulo: Manole; 2006. p. 288-307.

30. Humphereys AS, Thompson NJ, Miner KR. Intention to breastfeed in lowincome pregnant women: the role of social support and previous experience. Birth 1998;25:169-74.

31. Kong SK, Lee DT. Factors influencing decision to breastfeed. J Adv Nurs 2004;46:369-79.

32. Tohotoa J, Maycock B, Hauck YL, Howat P, Burns S, Binns CW. Dads make a difference: an exploratory study of paternal support for breastfeeding in Perth, Western Australia. Int Breastfeed J 2009;4:15.

33. Fletcher R, Vimpani G, Russell G, Keatinge D. The evaluation of tailored and web-based information for new fathers. Child Care Health Dev 2008;34:439-46.

34. Fernandes ER. Vivência do homem/pai no processo da amamentação do filho [tese de mestrado]. Natal: Universidade Federal do Rio Grande do Norte; 2003.

35. Rudman A, Waldenström U. Critical views on postpartum care expressed by new mothers. BMC Health Serv Res 2007;7:178.

36. Serafim D, Lindsey PC. Father's opinion about breast-feeding. Cienc Cuid Saude 2002;1:19-23.

37. Garfield CF, Isacco A. Fathers and the Well-Child Visit. Pediatrics 2006;117:e637-45.

38. Costa CR. Representação do papel do pai no aleitamento materno [tese de mestrado]. Distrito de Porto: Universidade do Porto; 2007.

39. Molina IB, Rojas PB. Knowledge, beliefs and attitudes of parents that have influence in the promotion and protection of breastfeeding [tese de mestrado]. Santiago: Pontificia Universidad Católica de Chile; 2000.

40. Brito RS, Enders BC, Soares VG. Breastfeeding: the contribution of the father Rev Bras Enferm 2005;19:105-12.

41. Shepherd CK, Power KG, Carter H. Examining the correspondence of breastfeeding and bottle-feeding couples' infant feeding attitudes. J Adv Nurs 2000;31:651-60.

42. Johansson K, Aarts C, Darj E. First-time parents' experiences of home-based postnatal care in Sweden. Ups J Med Sci 2010;115:131-7.

43. Earle S. Factors affecting the initiation of breastfeeding: implications for breastfeeding promotion. Health Promot Int 2002;17:205-14.

44. Khoury AJ, Mitra AK, Hinton A, Carothers C, Sheil H. An innovative video succeeds in addressing barriers to breastfeeding among low-income women. J Hum Lact 2002;18:125-31.

45. Iglesias Casás S. Sociocultural and healthcare factors influencing the choice and duration of breast feeding in a regional hospital. Enferm Clin 2008;18:142-6.

46. Susin LR, Giugliani ER. Inclusion of fathers in an intervention to promote breastfeeding: impact on breastfeeding rates. J Hum Lact 2008;24:386-92.

47. Kuan LW, Britto M, Decolongon J, Schoettker PJ, Atherton HD, Kotagal UR. Health system factors contributing to breastfeeding success. Pediatrics 1999;104:e28.

48. Cohen R, Lange L, Slusser W. A description of a male-focused breastfeeding promotion corporate lactation program. J Hum Lact 2002;18:61-5.

49. Wolfberg AJ, Michels KB, Shields W, O'Campo P, Bronner Y, Bienstock J. Dads as breastfeeding advocates: results from a randomized controlled trial of an educational intervention. Am J Obstet Gynecol 2004;191:708-12.

50. Stremler J, Lovera D. Insight from a breastfeeding peer support pilot program for husbands and fathers of Texas WIC participants. J Hum Lact 2004;20:417-22.

51. Rivera Alvarado I, Vázquez-García V, Dávila Torres RR, Parrilla Rodríguez AM. Exploratory study: breastfeeding knowledge, attitudes towards sexuality and breastfeeding, and disposition towards supporting breastfeeding in future Puerto Rican male parents. P R Health Sci J 2006;25:337-41. 